\title{
Low complexity PAPR reduction methods for carrier-aggregated MIMO OFDMA and SC-FDMA systems
}

\author{
Pochun Yen and Hlaing Minn ${ }^{*}$
}

\begin{abstract}
New generation wireless systems target to support much higher data rate which requires larger bandwidth than the existing systems. Lack of contiguous spectrum resources and the compatibility requirement with the legacy systems make the concept of carrier aggregation (CA) a practical means for supporting high data rate in next generation systems. One of the practical issues related to the CA is the dramatically increased peak-to-average power ratio of the time domain signal. This article proposes several low-complexity and no-overhead PAPR reduction methods for bandwidth aggregated systems with orthogonal frequency division multiplexing/multipleaccess or single-carrier frequency division multiple access in multi-in multi-out system configurations. For the proposed phase-rotation based PAPR reduction methods, a reliable phase rotation detector at the receiver is developed to maintain the receiver performance. Performance evaluation under 3GPP LTE-Advanced environment shows the advantage of the proposed methods while not requiring any signaling overhead.
\end{abstract}

\section{Introduction}

The demand of higher data rate to support various wireless services and applications increases dramatically over the last decade. 3GPP has adopted carrier aggregation (CA) [1] where multiple LTE Release 8 [2,3] component carriers (CCs) are combined to provide the targeted or required data rate. One of the downsides that CA systems face is the significantly increased peak-to-average power ratio (PAPR) of their time domain signal. Hence, PAPR control is an important practical issue for carrier aggregated systems.

There exist several approaches to address this PAPR problem (see $[4,5]$ for an overview) for orthogonal frequency division multiplexing (OFDM) systems. Some representative works resort to clipping and filtering [6-8], pulse shaping filters [9-11], and block coding [12-14] at the cost of either performance, complexity, or code rate. Several other schemes exploit multiple signal representations at the expense of complexity and signaling overhead. These schemes include partial transmit sequences (PTS) [15-17], selective mapping technique

\footnotetext{
* Correspondence: hlaing.minn@utdallas.edu

* Correspondence: hlaing.minn@utdallas.edu
Department of Electrical Engineering, University of Texas at Dallas, 800 W. Campbell Rd, Richardson, TX 75080, USA
}

(SLM) [18-22], tone reversion (TR) [23,24] and constellation expansion $[25,26]$.

However, all of those works consider typical OFDM systems which are not carrier aggregated systems. In addition, some systems (e.g., uplink (UL) of LTE Release 8 and LTE-A) use single carrier frequency division multiple access (SC-FDMA) which has different PAPR characteristics [27] than OFDM or OFDMA systems [28]. The promising schemes with significant PAPR reduction such as PTS and SLM require substantial signaling overhead which grows linearly with the number of CCs. The complexity of such schemes in the CA systems increases exponentially with the number of CCs. Thus, PAPR reduction methods with reduced complexity and lower or no signaling overhead are needed for CA systems.

In this article, we propose several PAPR reduction methods which aim to reduce system complexity, eliminate the need of signaling overhead and provide good PAPR reduction performance in carrier-aggregated OFDM/OFDMA and SC-FDMA multi input multi output (MIMO) systems. The first three methods namely multi-symbol SLM (MSSLM), partial SLM (PSLM) and sequential SLM (SSLM) are based on the SLM technique with some modifications. The fourth method called partial linear precoding across time (PLPT) relies on 
several different representations of the signal through linear precoding of subcarrier data across time, and the transmitter selects the representation with the lowest PAPR to transmit.

In a recent work [29] which considers a singleantenna OFDM system, signaling overhead associated with PAPR reduction is eliminated by means of inserting pilot tones in each OFDM symbol. The receiver obtains the phase rotations applied in the PAPR reduction from the inserted pilots. While applying a similar concept from [29] in our first three methods, we aim to reduce the cost of pilot tones at each OFDM symbol. We propose to use the pilots inserted for channel estimation and tracking according to the standard specification, thus eliminating requirement of additional pilots at each multiple access (MA) symbol, i.e., either OFDMA or SC-FDMA symbol. This leads to the use of PAPR optimization intervals with multiple symbol durations for the first three methods. Another difference from [29] is that we consider MIMO carrier-aggregated systems which have their own characteristics and constraints.

In the LTE-A, there are several MIMO schemes specified, including space frequency block coding (SFBC), spatial multiplexing (SM) and beam forming. The later two schemes require the modifications made on the signal of each antenna for PAPR reduction to be identical; otherwise, the original MIMO properties will be destroyed. Hence, in our proposed PAPR reduction schemes, all phase changes or linear precodings are made to be the same across all antennas for all MIMO schemes. This will limit the PAPR reduction capability, but the MIMO properties can be preserved. Our PAPR reduction methods can be applicable to multi-user MIMO scenario (also a mode of LTE-A downlink (DL)) as will be discussed later in the article.

Regarding PSLM, MSSLM and SSLM, while adopting SLM concept, our contributions include (i) the concept of multi-symbol PAPR optimization intervals for signaling overhead reduction, (ii) the concept of partial group selection for complexity reduction, (iii) adaptation to CA systems, (iv) the extension to the MIMO scenarios, and (v) a reliable phase rotation detector at the receiver to remove the effects of PAPR reducing phase rotations on the receiver performance. These methods provide low-complexity and require no signaling overhead by the exploitation of existing reference symbols for signaling overhead reduction. Parts of these contributions were presented in [30].

The fourth proposed method PLPT offers an alternative means of reducing PAPR by a linear precoding of data on some subcarriers across two adjacent OFDMA or SC-FDMA symbols. Like SLM based methods, every different representation of the same signal will have different PAPR value and the transmitter selects a representation which has the minimum PAPR to transmit. While LPT and SLM based methods share some contributions together, PLPT does not need multi-symbol PAPR optimization intervals, each of which contains pilot signal for signaling overhead reduction, because PLPT does not use phase rotation vectors. For PLPT, a modified receiver can detect whether the precoding has been applied or not.

The rest of the article is organized as follows. Section 2 presents system model, and Section 3 describes the proposed methods. Simulation results are discussed in Section 4, and Section 5 concludes this article.

\section{System model}

In this article, we assume a general cellular system which adopt OFDMA for its DL and use SC-FDMA (or discrete Fourier transform (DFT) spread OFDM) in its UL. The aggregation of several CCs is introduced in order to utilize wider bandwidth (for higher data rate) while keeping backward compatibility with the older standard. Each DL/UL transmission subframe for a user on a CC contains several subcarriers over several MA symbols forming a resource grid (RG).

Let $N_{t}, N_{1}$ and $N_{\text {symb }}$ denote the number of the transmit antennas, number of the MIMO layers and the subframe duration in unit of OFDM or SC-FDMA symbol duration, respectively. Define $f_{i}=f_{c_{i}}-f_{c_{0}}$ which represents the center frequency difference of the $i$ th $\mathrm{CC}$ and the reference 0th CC in the considered link (DL or UL). Figure 1 shows the baseband model of two CCs where the top CC is the reference 0th CC and the bottom CC is shifted to center frequency $f_{1}$. In general, there are two primary modes, SM and transmit diversity (TD) for DL. For UL, only SM mode is considered and a DFT precoding is applied between layer mapping and MIMO precoding stages.

\subsection{DL model}

In the DL MIMO SM scenario, the modulation symbols $\{d(n)\}$ are divided into multiple layers denoted as $\mathbf{b}_{i}(n)=\left[b_{i}^{(0)}(n), \ldots, b_{i}^{\left(N_{1}-1\right)}(n)\right]^{\mathrm{T}}$ over which a MIMO precoding is applied as

$$
\mathbf{Z}_{i}(n)=\mathbf{W b}_{i}(n)
$$

where $b_{i}^{(v)}(n)$ is the $n$th modulation symbol in the $v$ th layer of $i$ th CC and $\mathbf{W}$ is a $N_{\mathrm{t}} \times N_{\mathrm{l}}$ MIMO precoding matrix (see [2]). $\mathbf{Z}_{i}(n)=\left[z_{i}^{(0)}(n), \ldots, z_{i}^{\left(N_{\mathrm{t}}-1\right)}(n)\right]^{\mathrm{T}}$ is the vector of MIMO precoded symbols where $z_{i}^{(p)}(n)$ is the $n$th symbol of the antenna $p$.

For the DL MIMO TD scenario, the precoding for TD is defined for two or four antennas. For transmission on 


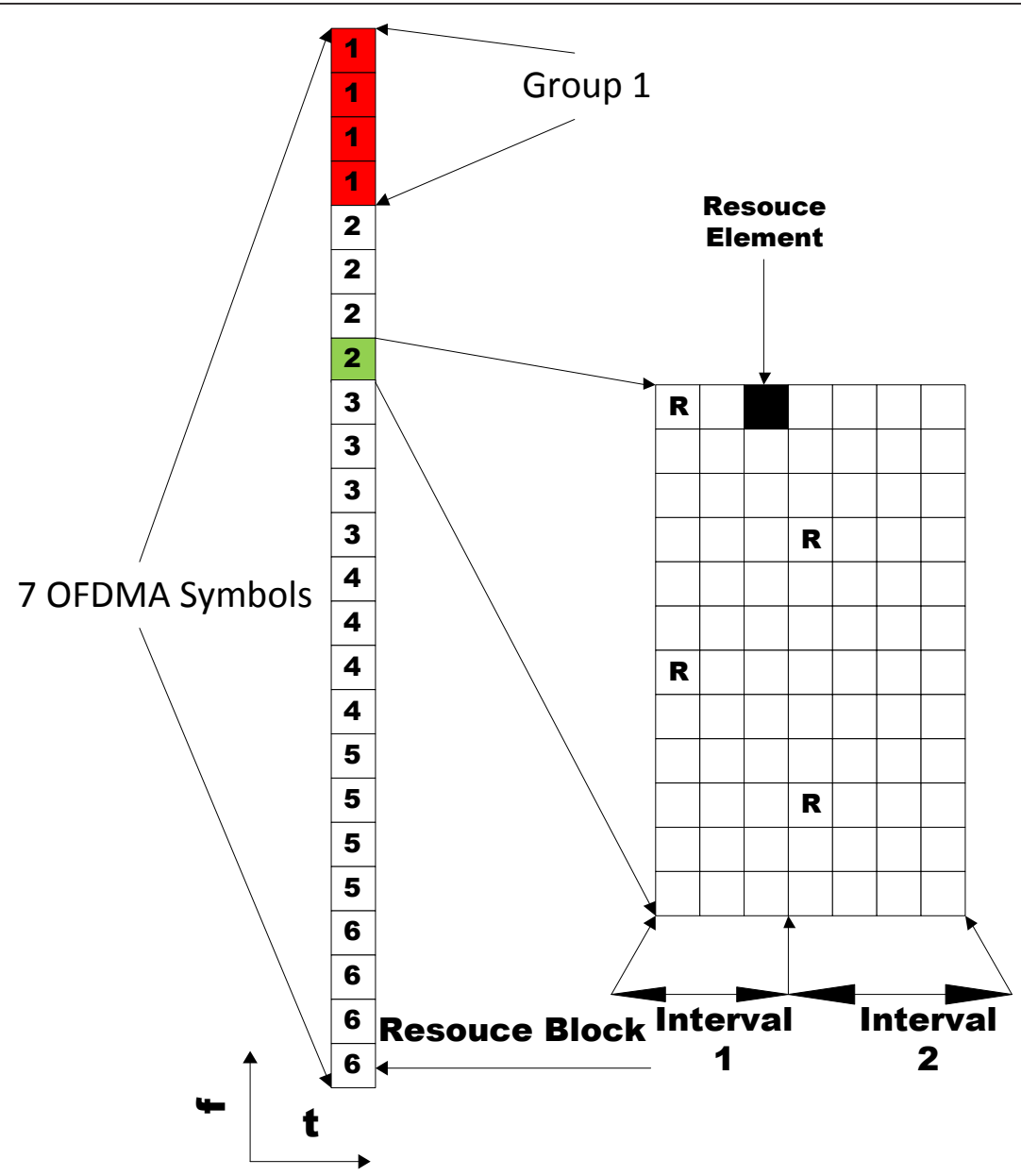

Figure 1 Baseband block diagram of the proposed methods (MSSLM, PSLM and SSLM) in UL scenario with 2 CCs in a single antenna setup. PAPR optimization is typically done in all discrete-time domain and in this case a larger IDFT size (where $U$ is the oversampling factor) should be used.

two antennas, space-frequency block code (SFBC) is adopted and the precoding operation is defined by

$$
\left[\begin{array}{c}
z_{i}^{(0)}(2 n) z_{i}^{(0)}(2 n+1) \\
z_{i}^{(1)}(2 n) z_{i}^{(1)}(2 n+1)
\end{array}\right]=\left[\begin{array}{cc}
b_{i}^{(0)}(n) & b_{i}^{(1)}(n) \\
-b_{i}^{(1)^{*}}(n) & b_{i}^{(0)^{*}}(n)
\end{array}\right] .
$$

In the four antennas scenario, a combination of the SFBC and the frequency-switched transmit diversity (FSTD) is implemented as

$$
\begin{gathered}
{\left[\begin{array}{l}
z_{i}^{(0)}(4 n) z_{i}^{(0)}(4 n+1) z_{i}^{(0)}(4 n+2) z_{i}^{(0)}(4 n+3) \\
z_{i}^{(1)}(4 n) z_{i}^{(1)}(4 n+1) z_{i}^{(1)}(4 n+2) z_{i}^{(1)}(4 n+3) \\
z_{i}^{(2)}(4 n) z_{i}^{(2)}(4 n+1) z_{i}^{(2)}(4 n+2) z_{i}^{(2)}(4 n+3) \\
z_{i}^{(3)}(4 n) z_{i}^{(3)}(4 n+1) z_{i}^{(3)}(4 n+2) z_{i}^{(3)}(4 n+3)
\end{array}\right]} \\
\quad\left[\begin{array}{cccc}
b_{i}^{(0)}(n) & b_{i}^{(1)}(n) & 0 & 0 \\
0 & 0 & b_{i}^{(2)}(n) & b_{i}^{(3)}(n) \\
-b_{i}^{(1)^{*}}(n) b_{i}^{(0) *}(n) & 0 & 0 \\
0 & 0 & b_{i}^{(3)^{*}}(n) b_{i}^{(2)^{*}}(n)
\end{array}\right] .
\end{gathered}
$$

\subsection{UL model}

For the UL MIMO scheme, after the modulation symbols are layered, each layer $b^{(v)}(n)$ will be DFT precoded as

$$
y_{i}^{(v)}(l R+k)=\frac{1}{R} \sum_{m=0}^{R-1} b_{i}^{(v)}(l R+m) e^{-j \frac{2 \pi m k}{R}}, \quad k=0, \ldots, R-1,
$$

where $R$ is the number of assigned subcarriers to the UL per user and $l$ ranges from 0 to $N_{\text {symb }}-1$ except those slots for the reference signal. In UL, only SM is adopted in the MIMO scheme; therefore, we can write the MIMO precoded symbols $\mathbf{Z}_{i}(n)$ in (1) as

$$
\mathrm{Z}_{i}(n)=\mathbf{W y}_{i}(n)
$$

where $\mathbf{y}_{i}(n)=\left[y_{i}^{(0)}(n), \ldots, y_{i}^{\left(N_{1}-1\right)}(n)\right]^{\mathrm{T}}$ is the vector of DFT precoded symbols.

For both DL and UL schemes, define $M_{\mathrm{sc}}\left(M_{\mathrm{sc}}=R\right.$ in $\mathrm{UL})$ as the number of subcarriers assigned in each CC, $\mathcal{F}_{i}$ as the set of subcarrier indices (which are in the 
increasing order) of the $i$ th $C C$ and $\mathcal{F}_{i}(m)$ represents the $m$ th index of $\mathcal{F}_{i}$. The final output $z_{i}^{(p)}(n)$ is mapped to the resource element $X_{i, l, k}^{(p)}$ of antenna $p$ as

$$
X_{i, l, k}^{(p)}=z_{i}^{(p)}\left(l M_{\mathrm{sc}}+m\right), \quad \text { for } k=\mathcal{F}_{i}(m)
$$

where $m=0, \ldots, M_{\mathrm{sc}}-1, l=0, \ldots, N_{\mathrm{symb}}-1$. The continuous-time baseband signal $s_{i, l}^{(p)}(t)$ in the $l$ th OFDMA or SC-FDMA symbol of the $i$ th $C C$ in a subframe of antenna $p$ is given by

$$
s_{i, l}^{(p)}(t)=\sum_{k \in \mathcal{F}_{i}} X_{i, l, k}^{(p)} \cdot e^{j 2 \pi k \Delta f t} e^{j 2 \pi f_{i} t},
$$

for $0 \leq t \leq N T_{s}$ (which will be shifted to the lth symbol interval later) and $l=0, \ldots, N_{\text {symb }}-1$, where $N$ is the inverse DFT (IDFT) size without oversampling and $T_{s}$ is the corresponding sample duration. Note that the lowpass equivalent representation uses $f_{c_{0}}$, the center frequency of the 0-th CC, as the reference bandpass carrier frequency in the conversion; hence the term $e^{j 2 \pi f_{i} t}$ appears in (7). We ignore cyclic prefix (CP) in the PAPR calculation since it is merely the cyclic extension of the signal itself.

\section{Proposed PAPR reduction methods}

\subsection{Multi-symbol selective mapping (MSSLM)}

In SLM, PAPR optimization is done for each OFDM symbol, therefore it requires high complexity and overhead. An intuitive approach for reducing complexity and overhead will be processing across multiple MA symbols, i.e., processing for PAPR reduction is not done at each symbol independently but rather applied the same across multiple symbols jointly. Therefore, we propose the concept of multi-symbol optimization interval denoted by the MA symbol index set $\mathcal{T}$. The choice of the PAPR optimization interval (the number of OFDM/ SC-FDMA symbols over which PAPR is defined and minimized) is constrained by the tradeoff between PAPR reduction capability and control signaling overhead. The optimization interval of one symbol provides the best PAPR reduction capability at the cost of substantial control signaling overhead. On the other hand, using the interval with a very large number of symbols will substantially save the signaling overhead but the PAPR reduction might be negligible or unjustifiable for the associated complexity. Thus, a proper tradeoff is necessary. Based on our investigation, we recommend a PAPR optimization interval of around four symbols for SC-FDMA based systems while for OFDM DL systems a slightly larger interval may be selected if associated PAPR reduction is acceptable.
As pilots or reference symbols are typically transmitted in practical systems, we can take advantage of them in removing or reducing the control signaling overhead. By including reference symbols in the optimization interval, i. e., applying the same phase rotations for both reference symbols and data symbols, the effects of the phase rotations can be absorbed in the channel estimates, or the phase rotation can be detected (see Section 3.5) and its effect can be removed at the receiver. By this way, no separate signaling overhead is required to inform the receiver of the applied phase rotations.

A transmission frame may contain more than one reference symbols. In this case, we may have more than one PAPR optimization intervals. To illustrate, let us consider the DL of the LTE-A system. There are seven symbols (normal CP length) in a slot and reference symbols for DL are placed at either first and fourth OFDMA symbols or at second and fifth OFDMA symbols. Considering the former, we can form two PAPR optimization intervals of three symbols and four symbols, with the corresponding symbol index sets $\mathcal{T}_{1}=\{0,1,2\}$ and $\mathcal{T}_{2}=\{3,4,5,6\}$, in each slot as shown in Figure 2. As there is one reference symbol in each interval, we do not need any control signaling overhead at all in this case.

With the introduction of the optimization interval, we propose MSSLM to overcome the complexity and overhead burden of the original SLM. Consider a PAPR optimization interval $\mathcal{T}$. All symbols $X_{i, l, k}^{(p)}$ of the IDFT input in the $i$ th $C C$ are partitioned into $S$ disjoint groups over the above optimization interval $\mathcal{T}$, represented by $\left\{\mathbf{X}_{i, l}^{(p)(q)}, q=0, \ldots, S-1\right\}$. Next, MSSLM applies phase rotations to all groups. The phase rotation $\varphi_{i, q}$ which rotates the $i$ th CC's $q$ th group is from the set $\Phi=\left\{\Phi_{m}: m=0, \ldots, P-1\right\}$, where $P$ is the number of phase choices applied to each group of subcarriers. Set $M$ as the number of CCs. We define a phase combination $\mathbf{v} \in \Phi^{\mathrm{SM}}$ which is a length-SM vector where its elements belong to $\boldsymbol{\Phi}$. Each different vector $\mathbf{v}$ refers to one set of possible phase rotations that can be applied onto the transmit signal. Therefore, the phase shifted signal corresponding to a phase combination $\mathbf{v}$, denoted by $\tilde{s}_{\mathrm{CA}}^{(p)}(\mathbf{v}, t)$, can be represented as

$$
\begin{aligned}
\tilde{s}_{\mathrm{CA}}^{(p)}(\mathbf{v}, t)= & \sum_{l \in \mathcal{T}} \sum_{i=0}^{M-1} \sum_{q=0}^{S-1} \sum_{k \in \mathcal{J}_{i, l, q}} \mathbf{X}_{i, l}^{(p)(q)} e^{j, \phi_{i, q}} e^{j 2 \pi\left(k \Delta f+f_{i}\right)\left(t-l N T_{s}\right)} \\
& g\left(t-l N T_{s}\right),
\end{aligned}
$$

where the set $\mathcal{J}_{i, l, q}$ contains indices of all subcarriers which belong to the $q$ th group of $i$ th $C C$ at $l$ th symbol. Note that we can set $\varphi_{0,0}$ to be zero without affecting the performance, thus the search space would be $\Phi^{\mathrm{SM}-1}$. Each 


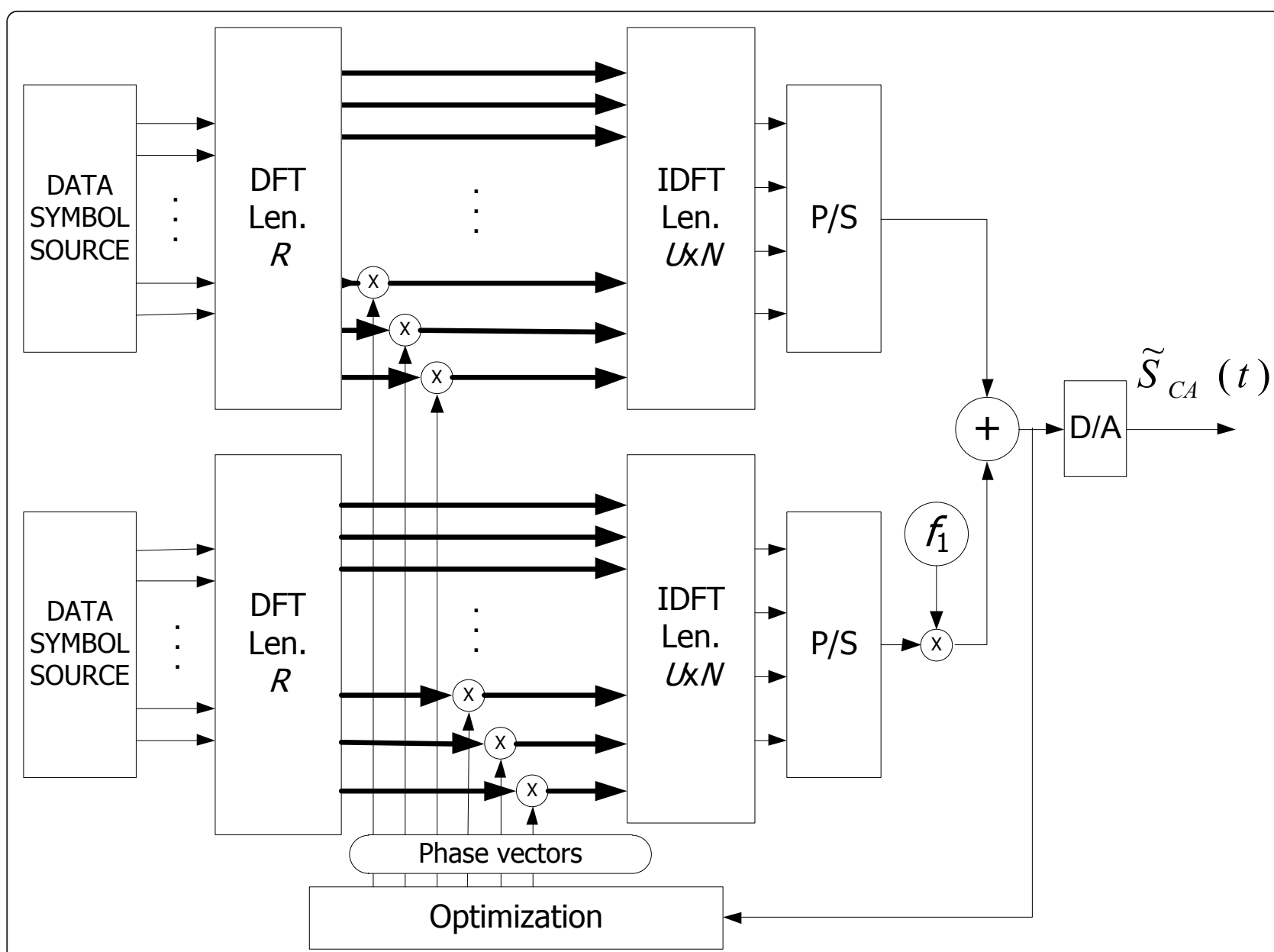

Figure 2 One DL OFDMA time slot where $R$ refer to reference symbol is illustrated with $S=6$ groups and each contains four resource blocks (48 subcarriers of 7 OFDMA symbols). Intervals 1 and 2 contain first 3 and later 4 OFDMA symbols respectively.

combination results in a different final PAPR value. The transmitter will simply choose the combination which provides the best result. Note that the PAPR reduction phase vectors are the same across antennas but the actual PAPR values are in general different from antenna to antenna. We need only one metric to decide which phase vector to be used for PAPR reduction. Denote $\mathbf{V}$ as the collection of all possible combinations vectors $\mathbf{v}$ where each vector represents one possible phase combination. We have tested two candidate metrics: (1) using the maximum PAPR across all antennas as

$$
\mathbf{v}^{*}=\arg \min _{\mathbf{v} \in \mathbf{V}} \max \left\{\operatorname{PAPR}\left[\tilde{s}_{\mathrm{CA}}^{(1)}(\mathbf{v}, t)\right], \ldots, \operatorname{PAPR}\left[\tilde{s}_{\mathrm{CA}}^{\left(N_{t}\right)}(\mathbf{v}, t)\right]\right\}
$$

and (2) using the average PAPR across all antennas as

$$
\mathbf{v}^{*}=\arg \min _{\mathbf{v} \in \mathbf{V}} \frac{1}{N_{t}} \sum_{\mathrm{p}=0}^{N_{t}-1} \operatorname{PAPR}\left[\tilde{s}_{\mathrm{CA}}^{(p)}(\mathbf{v}, t)\right]
$$

where

$$
\operatorname{PAPR}\left[\tilde{s}_{\mathrm{CA}}^{(p)}(\mathbf{v}, t)\right]=\frac{\max \left\|\tilde{s}_{\mathrm{CA}}^{(p)}(\mathbf{v}, t)\right\|^{2}}{\mathrm{E}\left[\left\|\tilde{s}_{\mathrm{CA}}^{(p)}(\mathbf{v}, t)\right\|^{2}\right]} .
$$

We have found out that using the latter provides a better result, thus we have adopted it in our methods. Therefore, we can write the time-domain transmit signals which have the minimum PAPR as

$$
\tilde{s}_{\mathrm{CA}}^{(p) *}(t)=\tilde{s}_{\mathrm{CA}}^{(p)}\left(\mathbf{v}^{*}, t\right), \quad \text { for } p=0, \ldots, N_{\mathrm{t}-1} .
$$

\subsection{Partial selective mapping (PSLM)}

In MSSLM, since all $S$ groups are selected for phase optimization, the complexity increases exponentially with $S$. To be practical, $S$ will be limited to a small number. Alternatively, we propose PSLM which applies 
phase optimization only to part of all groups. Like MSSLM, All symbols $X_{i, l, k}^{(p)}$ of the IDFT input in the $i$ th CC are partitioned into $S^{\prime}$ disjoint groups over the optimization interval $\mathcal{T}$ where $S$ ' can be larger $S$. Next, PSLM applies phase rotations to $K$ selected groups out of all $S^{\prime}$. Note that the phase rotations $\left\{\phi_{i, q^{\prime}}\right\}$ for unselected groups are set to zero.

The total number of groups per CC, $S^{\prime}$, as well as the number of selected groups for processing per CC, $K$, provide a tradeoff between PAPR reduction capability and complexity (plus overhead) if no reference or pilot symbols are available. The underlying system parameters (e.g., resource block sizes) also shape the choice of those group numbers.

The other aspect that should be considered is how to address for different resource block sizes especially for UL. In order to limit the complexity regardless of the resource block size, we adopt to use a fixed number of groups while adjusting the size of each group to accommodate for the change in the resource block size.

The next step is to find out which groups should be selected. For example, in the LTE-A scenario with four selected groups out of six groups, there are 15 possible selections and it is unlikely that all will give the same PAPR reduction performance. As will be shown later in Section 4, a proper selection does yield a better PAPR reduction performance.

As DL contains data for all users, it has much larger resource size than the UL. The same procedure can be applied for DL but the values of $S^{\prime}$ and $K$ can be chosen differently (especially, larger than UL case to gain more PAPR reduction) if desired. On the other hand, base stations typically have power amplifiers with large linearity range and energy consumption is of less concern. Hence, even using the same values of $S^{\prime}$ and $K$ as in the UL may be sufficient for the DL.

Note that all the steps for the partial group selection can be done offline once, and hence there is no implementation complexity associated with it. The group numbers and selection are fixed across all CCs of all antennas. This provides simplicity and modularity in implementation.

With the concept of optimization interval and partial group selection, the partially phase shifted signal $\tilde{s}_{\mathrm{CA}}^{(p)}(\mathbf{v}, t)$ corresponding to a phase combination $\mathbf{v} \in$ $\Phi^{K M}$ for PSLM can be written as

$$
\begin{aligned}
\tilde{s}_{\mathrm{CA}}^{(p)}(\mathbf{v}, t)= & \sum_{l \in \mathcal{T}} \sum_{i=0}^{M-1} \sum_{q^{\prime}=0}^{S^{\prime}-1} \sum_{k \in \mathcal{J}_{i, l, q^{\prime}}} \mathbf{X}_{i, l}^{(p)\left(q^{\prime}\right)} e^{j \phi_{i, q^{\prime}}} e^{j 2 \pi\left(k \Delta f+f_{i}\right)\left(t-l N T_{s}\right)} \\
& g\left(t-l N T_{s}\right),
\end{aligned}
$$

where $q^{\prime}=0, \ldots, S^{\prime}-1$ and the selection of signal which has the minimum PAPR is similar to (12). The detection of the phase rotations can be done at the receiver in a similar manner as in MSSLM, and hence no signaling overhead is required for PAPR reduction.

\subsection{Sequential selective mapping (SSLM)}

This scheme aims to reduce the complexity of SLMbased approaches by adopting a sequential optimization. Define the phase combination set $\mathbf{V}_{r}^{\prime}$ which contains all length-S' $M$ vectors as

$$
\mathbf{v}_{r}^{\prime}=\left[\phi_{0,0}^{(r)}, \phi_{0,1}^{(r)}, \ldots, \phi_{0, S^{\prime}-1}^{(r)}, \phi_{1,0}^{(r)}, \ldots, \phi_{i, q}^{(r)}, \ldots, \phi_{M-1, S^{\prime}-1}^{(r)}\right]
$$

where its $(r+1)$ th to $S^{\prime} M$ th elements are zeros (i.e. $\stackrel{(r)}{\left\lfloor(r+1) / S^{\prime}\right\rfloor,(r+1) \bmod S^{\prime}}$ to $\phi_{M-1, S^{\prime}-1}^{(r)}$ are all zeros), first to $(r-1)$ th elements are fixed by the previous $(r-1)$ searches and the $r$ th element $\phi_{\left\lfloor r / S^{\prime}\right\rfloor, r \bmod S^{\prime}}^{(r)}$ is the only variable to be optimized at the $r$ th search. Alternatively, we can define $\mathbf{v}_{r}^{\prime}$ as

$$
\mathbf{v}_{r}^{\prime}=\left[\phi_{0,0}^{(r)}, \phi_{1,0}^{(r)}, \ldots, \phi_{M-1,0}^{(r)}, \phi_{0,1}^{(r)}, \ldots, \phi_{i, q}^{(r)}, \ldots, \phi_{M-1, S^{\prime}-1}^{(r)}\right]
$$

which yields the same PAPR reduction characteristics based on our investigation results. In fact, we can set $\varphi_{0,0}$ to be zero without affecting the PAPR reduction performance, thus we can start from $r=2$.

In SSLM, we can represent the sequentially phase shifted signal $\tilde{s}_{\mathrm{CA}}^{(p)}\left(\mathbf{v}_{r}^{\prime}, t\right)$ at the $r$ th iteration as

$$
\begin{aligned}
\tilde{s}_{\mathrm{CA}}^{(p)}\left(\mathbf{v}^{\prime}{ }_{r}, t\right)= & \sum_{l \in \mathcal{T}} \sum_{i=0}^{M-1} \sum_{q=0}^{S^{\prime}-1} \sum_{k \in \mathcal{J}_{i, l, q}} \mathbf{X}_{i, l}^{(p),(q)} e^{j \phi_{i, q}^{(r)}} e^{j 2 \pi\left(k \Delta f+f_{i}\right)\left(t-l N T_{s}\right)} \\
& g\left(t-l N T_{s}\right),
\end{aligned}
$$

where $r=2, \ldots, S^{\prime} M$. For every $r$, there are $P$ possible phases to apply, therefore, $P\left(S^{\prime} M-1\right)$ combinations of phase rotations are available to apply to the RGs differently. At the $r$ th iteration, the selected combination $\mathbf{v}_{r}^{*}$ is given by

$$
\mathbf{v}_{r}^{\prime *}=\arg \min _{\mathbf{v}_{r}^{\prime} \in \mathbf{V}_{r}^{\prime}} \frac{1}{N_{t}} \sum_{\mathrm{p}=1}^{N_{t}} \operatorname{PAPR}\left[\tilde{s}_{\mathrm{CA}}^{(p)}\left(\mathbf{v}_{r}^{\prime}, t\right)\right]
$$

where the definition of the PAPR is given by (11). The search continues in an increasing order of $r$ until a predefined PAPR threshold PAPR threshold is reached by $\tilde{s}_{\mathrm{CA}}\left(\mathbf{v}_{r}^{\prime *}, t\right)$ where

$$
\operatorname{PAPR}\left[\tilde{s}_{\mathrm{CA}}^{(p)}\left(\mathbf{v}_{r}^{*}, t\right)\right] \leq \operatorname{PAPR}_{\text {threshold }}
$$


for all antennas or until $r=S^{\prime} M$. The phase rotation effect at the receiver can be addressed in the same way as in MSSLM, thus requiring no associated signaling overhead.

\subsection{Partial linear precoding across time (PLPT)}

This scheme aims to remove the requirement of explicit signaling (e.g., SLM) or pilot-based implicit signaling (e.g., MSSLM, SSLM and PSLM), thus providing overhead saving and flexibility in its application. It applies linear precoding of data of some subcarrier groups across every two MA symbols. The choice of which subcarrier groups is based on the minimum average PAPR as defined in (10). Denote $\mathbf{w}$ to be the group indices selected to be PLPT-processed and $r$ be the index of the MA symbol pair corresponding to the MA symbol index $l=2 r$ and $2 r+1$. After the PLPT process, the subcarrier data of the $r$ th MA symbol pair are given by

$$
\left[\begin{array}{l}
\mathbf{X}_{i, 2 r}^{(p)(q)} \\
\mathbf{X}_{i, 2 r+1}^{\prime(p)(q)}
\end{array}\right]=\mathbf{P}_{i, r}^{(p)(q)}\left[\begin{array}{l}
\mathbf{X}_{i, 2 r}^{(p)(q)} \\
\mathbf{X}_{i, 2 r+1}^{(p)(q)}
\end{array}\right]
$$

where

$$
\mathbf{P}_{i, r}^{(p)(q)}=\frac{1}{\sqrt{2}}\left[\begin{array}{cc}
1 & 1 \\
1 & -1
\end{array}\right], \quad \text { if }(i, q) \in \mathrm{w}
$$

and

$$
\mathbf{P}_{i, r}^{(p)(q)}=\left[\begin{array}{ll}
1 & 0 \\
0 & 1
\end{array}\right], \quad \text { otherwise }
$$

The corresponding time domain signal over two MA symbol interval can be written as

$$
\begin{gathered}
\tilde{s}_{\mathrm{CA}}^{(p)}(\mathbf{w}, t)=\sum_{l=2 r}^{2 r+1} \sum_{i=0}^{M-1} \sum_{q=0}^{S^{\prime}-1} \sum_{k \in \mathcal{J}_{i, l, q}} \mathbf{X}_{i, l}^{\prime(p)(q)} e^{j 2 \pi\left(k \Delta f+f_{i}\right)\left(t-l N T_{s}\right)} \\
\quad g\left(t-l N T_{s}\right) .
\end{gathered}
$$

Similar to (12), the signal transmitted is the one with the optimal selection $\mathbf{w}^{*}$ which yields the lowest average PAPR. An illustration of PLPT method is shown in Figure 3. PLPT does not require any reference signal to convey the information of the selection made by the transmitter. At the receiver, whether PLPT is applied on a selected group or not can be determined from the equalized received signals on those subcarriers. Denote those equalized signals corresponding to $\left\{X_{i, 2 r}^{\prime(p)(q)}, X_{i, 2 r+1}^{\prime(p)(q)}\right\}$ by $\left\{Y_{i, 2 r}^{\prime(p)(q)}, Y_{i, 2 r+1}^{\prime(p)(q)}\right\}$. Then, the receiver can apply the same LPT processing on those equalized signals as

$$
\left[\begin{array}{l}
\hat{\mathbf{X}}_{i, 2 r}^{(p)(q)} \\
\hat{\mathbf{X}}_{i, 2 r+1}^{(p)(q)}
\end{array}\right]=\left[\mathbf{P}_{i, r}^{(p)(q)}\right]^{T}\left[\begin{array}{c}
\mathbf{Y}_{i, 2 r}^{(p)(q)} \\
\mathbf{Y}_{i, 2 r+1}^{\prime(p)(q)}
\end{array}\right]
$$

to get estimates of the original signal before precoding at the transmitter. Alternatively, the receiver can perform maximum likelihood (ML) detection over a larger signal space consisting of both LPT-processed and unprocessed symbols.

\subsection{Phase rotation detection}

Reference signal aids in acquiring and tracking channel knowledge for data detection at the receiver. The use of phase rotations for PAPR reduction over multiple intervals within a transmission frame should not affect the channel estimation and tracking. This issue can easily be addressed by detecting the phase rotations at the receiver based on the reference signal. There can be many ways to find out the phase rotations at the receiver. Here we provide a simple solution. For each reference signal on each $\mathrm{CC}$ at each receive antenna $m$, consider the same group partitions as at the transmitter with $\mathcal{J}_{q}$ and $\mathcal{L}^{(q, l)}$ denoting the subcarrier indexes of group $q$ and of pilots for transmit antenna $l$ in group $q$, respectively. Note that $\mathcal{L}^{(q, l)}$ is different for different $l$ as in LTE-A. Suppose the phase rotation applied for PAPR reduction at transmit antenna $l$ is $\varphi_{q}$ (same for all $l$ ). Then the received reference signal $Y_{m}(k)$ and the original reference signal $R_{l}(k)$ on pilot tone $k \in \mathcal{L}^{(q, l)}$ are related by

$$
Y_{m}(k)=H_{l, m}(k) e^{j \phi q} R_{l}(k)+N_{m}(k), \quad k \in \mathcal{L}^{(q, l)}
$$

where $H_{l, m}(k)$ is the channel gain on subcarrier $k$ corresponding to transmit antenna $l$ and receive antenna $m$ and $N_{m}(k)$ is the noise term on tone $k$ at receive antenna $m$.

The channel estimate $\hat{\tilde{H}}_{l, m}(k)$ at pilot location which includes the effect of phase rotation can be easily obtained as

$$
\hat{\tilde{H}}_{l, m}(k)=\frac{Y_{m}(k)}{R_{l}(k)}=H_{l, m}(k) e^{j \phi_{q}} R_{l}(k)+\frac{N_{m}(k)}{R_{l}(k)}, \quad k \in \mathcal{L}^{(q, l)} .
$$

For each group $q$, based on $\left\{\hat{\tilde{H}}_{l, m}(k): k \in \mathcal{L}^{(q, l)}\right\}$, the estimates of phase-rotated channel gains on all tones within the group $q$ denoted by $\left\{\hat{\tilde{H}}_{l, m}(k): k \in \mathcal{J}_{q}\right\}$ are obtained by interpolation/extrapolation (we use cubic interpolation). Note that the last subcarrier of group $q$ denoted by $q_{0}$ and the first subcarrier of group $q+1$ denoted by $q_{1}$ are adjacent and hence the channel gains on these two tones will be almost the same. Once all groups have been processed for all transmit-receive 


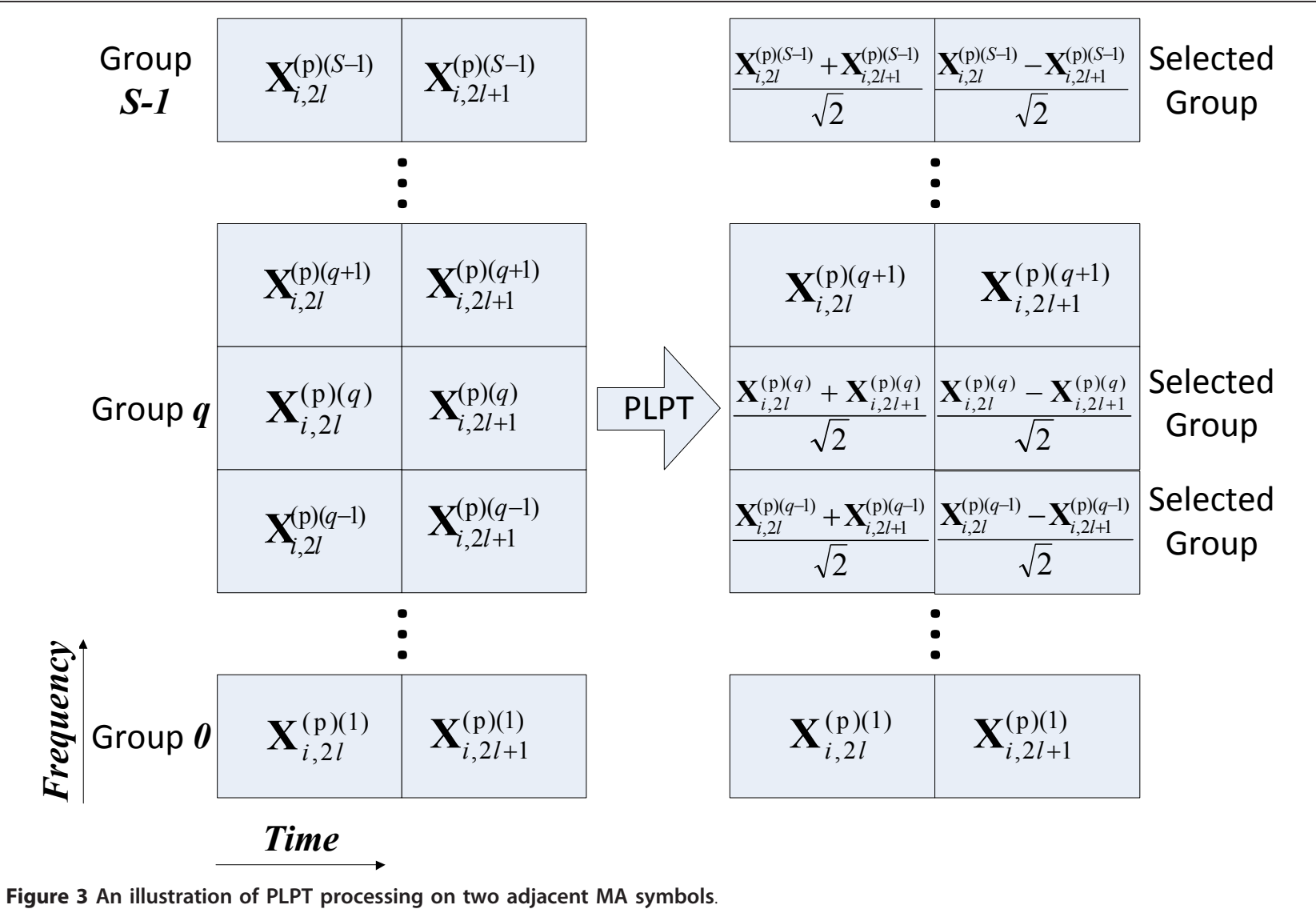

antenna pairs, the phase difference $\hat{\Delta} \phi^{(q),(q+1)}$ between $\varphi_{q}$ and $\varphi_{q+1}$ can be obtained from the estimates of phase-rotated channel gains on $q_{0}$ and $q_{1}$ as

$$
\hat{\Delta} \phi^{(q),(q+1)}=\left\langle\text { angle }\left\{\sum_{l, m} \hat{\tilde{H}}_{l, m}\left(q_{1}\right) \hat{\tilde{H}}_{l, m}^{*}\left(q_{0}\right)\right\}\right\rangle
$$

where $\langle\cdot\rangle$ represents a slicer which maps the angle in the argument to the nearest phase from the possible phase rotations. To retrieve $\left\{\varphi_{q}\right\}$ from $\left\{\hat{\Delta} \phi^{(q)},(q+1)\right\}$, a reference phase is needed. For MSSLM and SSLM, the phase of the first group of each $\mathrm{CC}$ can be set to zero and used as the phase reference. For PSLM, as some of the groups are not phase-rotated, their group phases are zero and can be used as the phase reference.

Once the phase rotations have been known at the receiver, the estimates of the channel gains without phase rotation can easily be obtained by simply removing the phase rotation effects from $\left\{\hat{\tilde{H}}_{l, m}(k)\right\}$. Next, further improvement of channel estimates (e.g. by exploiting limited channel delay spread) or/and channel tracking for time-varying channels can be done as in conventional systems. Thus, with reliable phase rotation detection as will be corroborated in the simulation section, the performance of the remaining receiver processing algorithms will not be affected.

\subsection{Applicability to multi-user MIMO}

In multi-user MIMO (MU-MIMO), data streams of multiple users occupy the same spectrum. In LTE-A DL, MUMIMO mode supports two simultaneous users on the same set of subcarriers. MU-MIMO uses additional user specific pilot sequences so that MIMO channels of different users can be distinguished at each user. Any processing for PAPR reduction should not disturb the relationship among different user specific pilot sequences.

For the proposed MSSLM, PSLM, and SSLM methods, the size of a group is 18 resource blocks (i.e., 216 subcarriers). If the users of MU-MIMO mode occupy the resource blocks within a group of the PAPR reduction approach, then the phase rotation of the proposed methods will be the same for all subcarriers assigned to those users. Then there will be no effect on the interrelationship of user specific pilot sequences and the proposed methods can be applied to the MU-MIMO scenario. The required 
condition for the applicability can easily be met by means of an appropriate resource allocation algorithm.

The PLPT method can be applicable to MU-MIMO scenario as follows. When we apply LPT processing on a group, we can skip LPT processing on the tones which contain user specific pilot sequences. Then those user specific pilot sequences are untouched, and MU-MIMO transmission is maintained. In this case, the resource blocks assigned to MU-MIMO users need not be limited within a group of the PAPR reduction approach.

\section{Performance results and discussion}

\subsection{System setup}

System parameters are adopted from the current LTE-A specification up to date [1] where $N_{\mathrm{sc}}=1320$ and $\Delta f=15$ $\mathrm{kHz}$. The oversampling factor for PAPR calculation is $U=$ 8 , and hence IDFT size of each CC is $N \times U=2,048 \times 8$ $=16,384$ for the reliable computation of PAPR. The predefined phase set is $\boldsymbol{\Phi}=\{0, \pi\}$ for PSLM and MSSLM. We use $\Phi=\left\{0, \frac{\pi}{2}, \pi, \frac{3 \pi}{2}\right\}$ for SSLM because of its low complexity. Note that, unlike MSSLM and PSLM, in SSLM, the complexity increases only linearly with a larger $P$. To evaluate MIMO CA systems without complexity burden, we use two non-contiguous CCs (300 MHz apart) and $N_{\mathrm{t}}$ = 2 antennas for both UL and DL system. We expect that the use of larger number of CCs would give larger PAPR reduction. Number of groups $S^{\prime}=6$ for PSLM, SSLM and PLPT and the number of selected groups $K=4$ for both PSLM and PLPT. MSSLM uses $S=K=4$ groups in each CC and SSLM uses all 6 groups without setting a PAPR threshold. The optimization interval for MSSLM, PSLM and SSLM is set to four symbols and PLPT uses a pair of MA symbols as its optimization interval. For the UL where SC-FDMA is used, the considered UL user occupies $R=72$ subcarriers (contiguous) on each CC. In DL OFDM scenario, we assume 1,296 subcarriers are occupied by multiple users on each CC, where in LTE 1296 is the largest number that a base station can allocate to a user.

In evaluating PAPR reduction techniques, complementary cumulative distribution function (CCDF) of PAPR is commonly used. Fran et al. [27] reported a linear dependency between average PAPR and required power backoff for the range of power backoff up to $9 \mathrm{~dB}$. On the other hand, [26,31] proposed that CCDF of instantaneous normalized power (INP) conveys a more relevant measure. Here, we will present both performance metrics. To have a fair comparison among different methods, in each result, PAPR calculation is done based on each MA symbol for each antenna.

\subsection{PAPR performance}

First, we evaluate the effect of group selection in LTE-A UL SISO scenario. 72 subcarriers are divided into 6 groups. We select 4 out of all 6 groups and all 15 possible selections are evaluated by simulation, and some selected results in terms of the CCDF of PAPR are shown in Figure 4. The CCDF curve of "Original" represents the time domain signal without any PAPR reduction method applied. Selection 1 curve represents the case where the four phase-rotated groups are located at the last four group locations. Selection 2 curve corresponds to the $2-5$ th group locations, while selection three curve chooses the first four group locations.

The results show that the group selection can make a difference in PAPR reduction. The group selection 1 shows the best performance and hence it is adopted in the rest of the simulations for PSLM and PLPT.

The CCDF performances for the UL MIMO SC-FDMA are shown in Figure 5. Note that the choice of SC-FDMA for UL is due to its low PAPR characteristics, yet the proposed techniques enable additional PAPR reduction gains. The MSSLM provides about 1.2 dB PAPR reduction over the original signal, while the PSLM achieves about 1.5 dB PAPR reduction at a CCDF level of 0.01 . PLPT has better performance than PSLM, achieving about $2 \mathrm{~dB}$ PAPR reduction. SSLM performs similarly to MSSLM, however, it requires less complexity. These gains translate into better energy efficiency for handheld mobile devices. Figure 6 shows the CCDF of INP. As it conveys sample-wise average performance, the gaps between different curves are much reduced if compared to the PAPR curves in Figure 5. Noticeably, PLPT method outperforms all other methods.

The DL MIMO OFDMA results are shown in Figure 7. Although base stations can handle much larger PAPR than mobile devices, the use of multiple CCs results in relatively large PAPR even for the base stations as can be seen in the figure with only two CCs. PLPT reduces PAPR by $2.1 \mathrm{~dB}$ at a CCDF level of 0.01 while PSLM has $1.8 \mathrm{~dB}$ in PAPR reduction. When MSSLM produces 1.6 dB PAPR reduction, the low complexity SSLM decreases PAPR by $1.5 \mathrm{~dB}$. Similarly, we provide Figure 8 to show the CCDF of INP of a time domain sample. The same discussion as for Figure 6 applies here as well.

We tested the performance of our proposed phase rotation detection for the DL $2 \times 2$ MIMO setup described above with four possible phases at SNR of $3 \mathrm{~dB}$. Out of the results for 10,000 independent channel realizations, we observed no phase rotation detection error. This corroborates reliable performance of the proposed phase rotation detector and confirms the applicability of the proposed phase-rotation-based PAPR reduction methods without affecting the receiver performance.

\subsection{Complexity and overhead}

The PAPR reduction performance of original SLM will be better than the proposed methods as it applies PAPR 


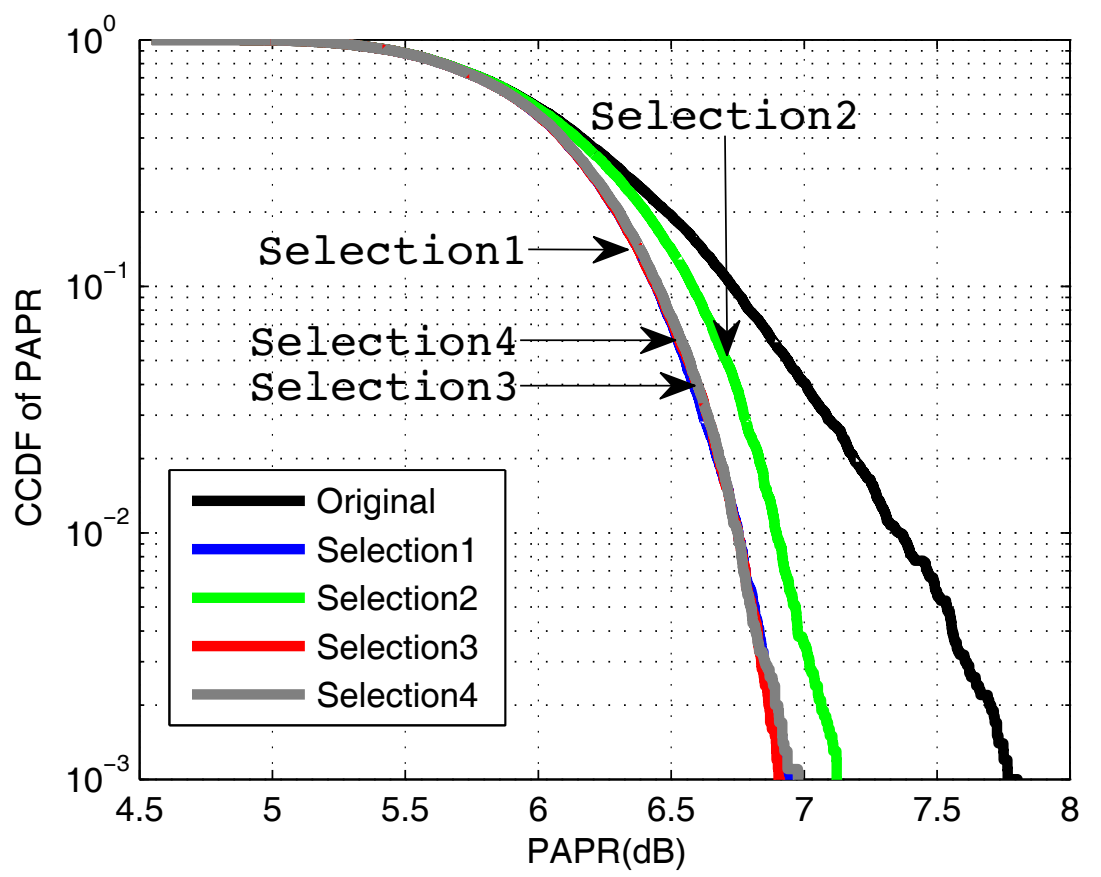

Figure 4 Effect of group selection on PAPR in UL SC-FDMA with QPSK modulation, 1 CC, single Antenna, obtained by 10,000 MonteCarlo simulations.

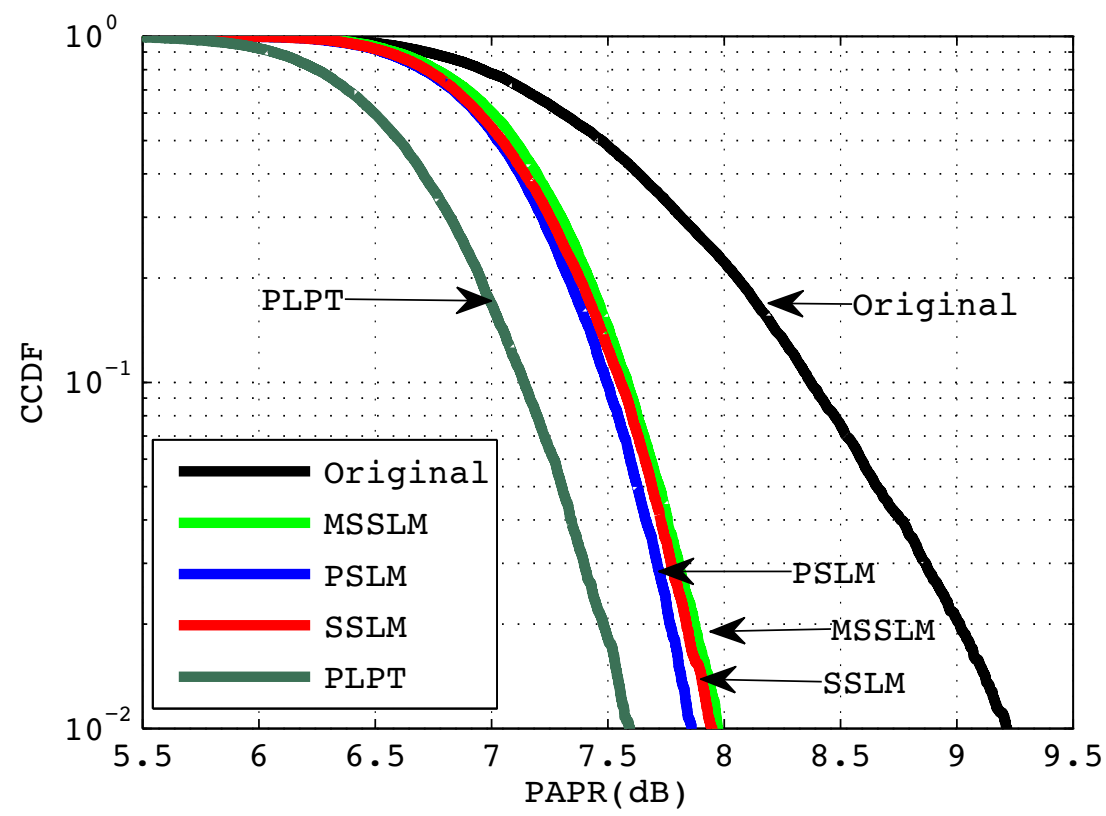

Figure 5 PAPR characteristics of the proposed schemes in UL MIMO SC-FDMA with 16-QAM modulation, 2 CCs, 2 antennas, obtained by 10,000 Monte-Carlo runs. 


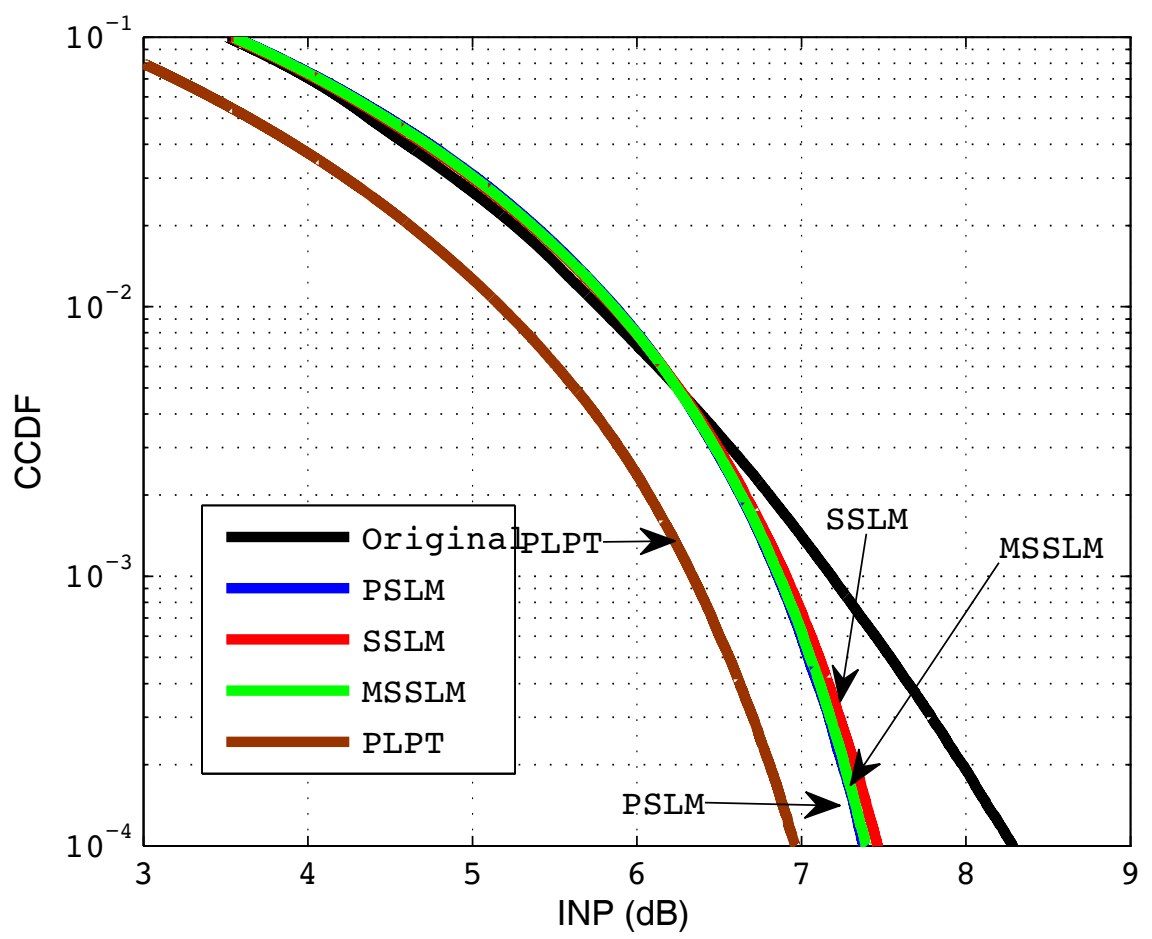

Figure 6 The characteristics of INP of a time domain sample of the proposed schemes in UL MIMO SC-FDMA, 16-QAM modulation, 2CCs, 2 antennas.

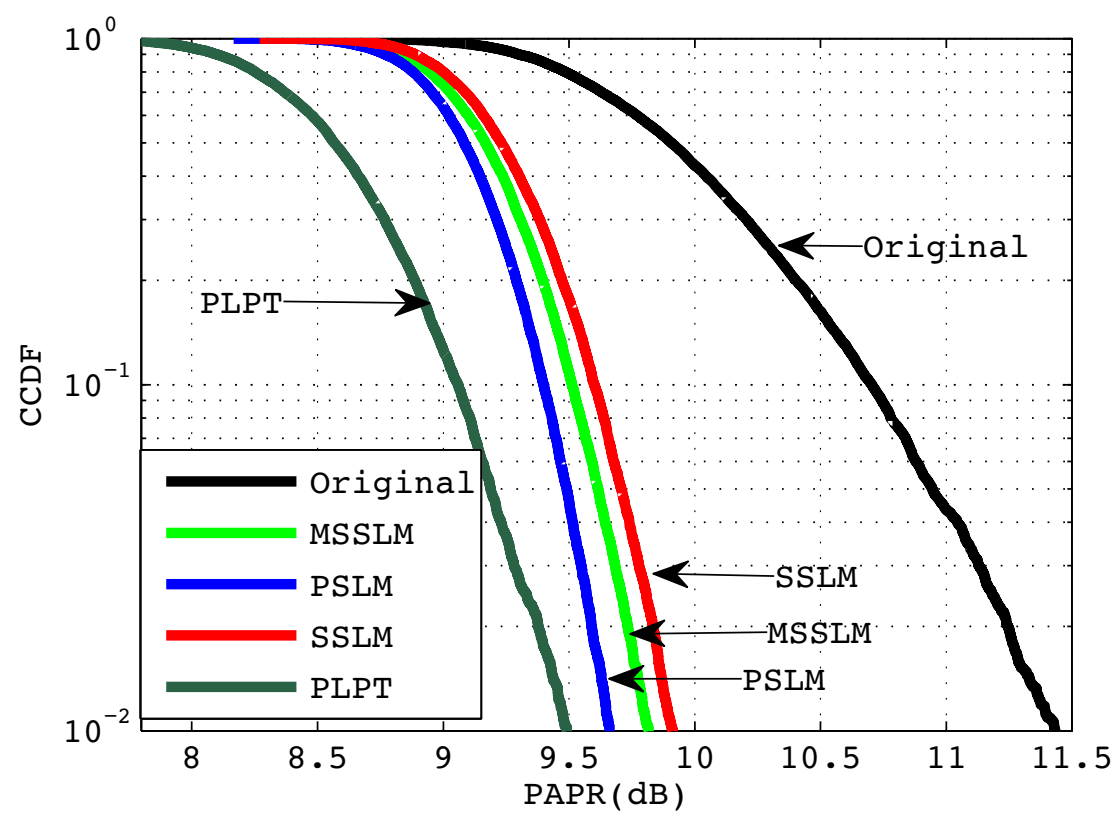

Figure 7 PAPR characteristics of the proposed schemes in DL MIMO OFDMA, 16-QAM modulation, 2CCs, 2 antennas, obtained by 10,000 Monte-Carlo runs. 


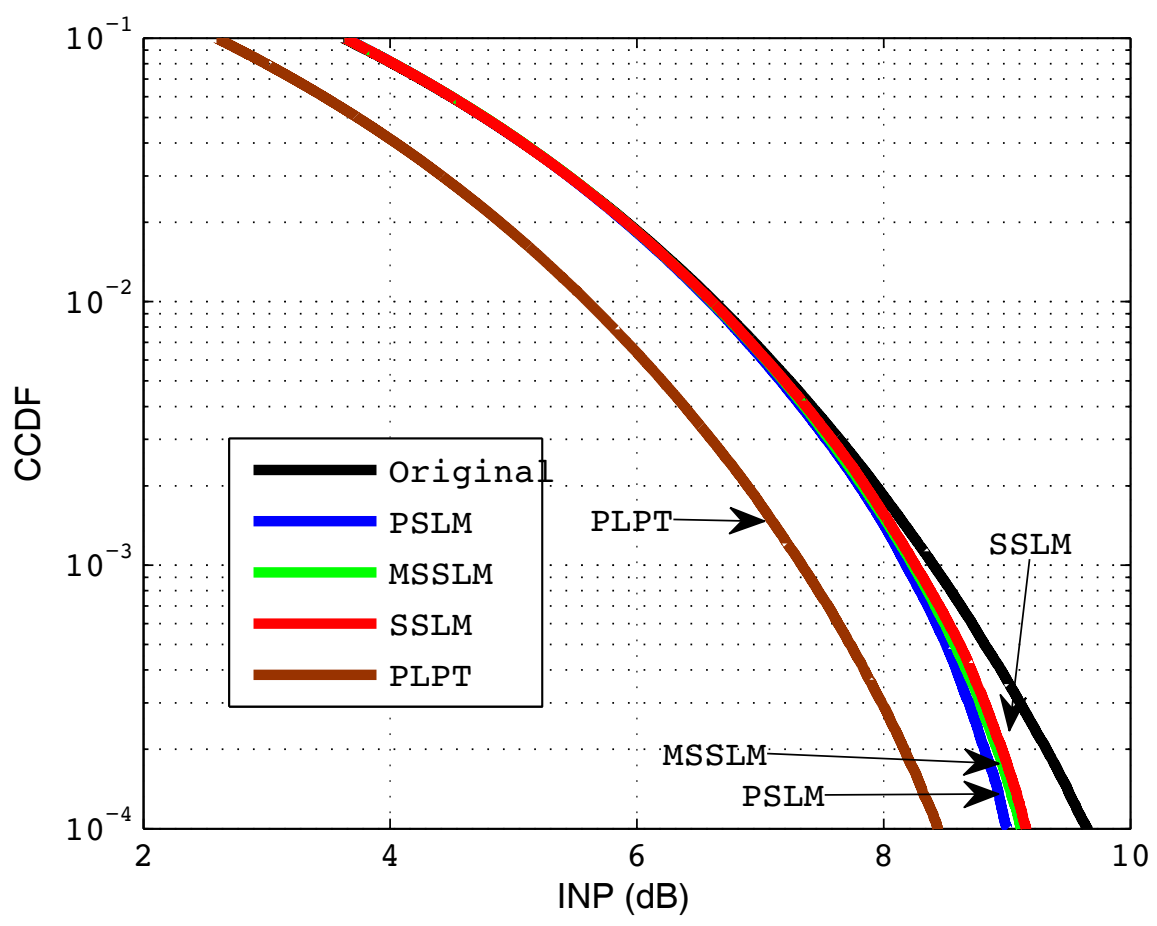

Figure 8 The characteristics of INP of a time domain sample of the proposed schemes in DL MIMO OFDMA, 16-QAM modulation, $2 \mathrm{CCs}, 2$ antennas.

reduction on each symbol. However, it is too complex to simulate with the aforementioned simulation parameters, and it requires substantial signaling overhead while the scope of the proposed methods stays within the framework with low complexity and no signaling overhead. If the original SLM [18] were applied for UL scenario, it would require $M \times N_{\text {symb }} \times P^{\mathrm{SM}}=2 \times 4 \times$ $2^{6 \times 2}=32,768$ IDFT operations with IDFT size $N \times U$ $=16,384, N_{\text {symb }} \times P^{\mathrm{SM}}=4 \times 2^{6 \times 2}=16,384$ PAPR calculations, each based on 16,384 samples, and $N_{\text {symb }} \times$ $\left\lceil\log _{2} P^{\mathrm{SM}}\right\rceil=48$ bits (not coded yet for error protection) for control overhead. Hence it is not practically appealing due to its high complexity and overhead. On the other hand, PSLM use 512 IDFT operations with IDFT size 16,384, and 256 PAPR calculations based on 16384 $\times 4=65,536$ samples when using optimization interval of four symbols. PLPT requires 512 IDFT operations with IDFT size 16, 384, and 256 PAPR calculations based on $16,384 \times 2=32$, 768 samples for every two MA symbols and additional processing at the receiver side to detect the presence of precoding. MSSLM use SM - 1 and SSLM use $S^{\prime} M-1$ groups. Fixing $\varphi_{0,0}$ to 0 will not affect their PAPR characteristics. Therefore, MSSLM uses $M P^{S M-1}=256$ IDFT operations with IDFT size 16,384 , and $P^{S M-1}=128$ PAPR calculations based on $16,384 \times 4=65,536$ samples when using optimization interval of four symbols. SSLM only requires $P M\left(S^{\prime} M-1\right)=88$ IDFT operations (note: $P$ is four for SSLM) with IDFT size 16, 384, and $P\left(S^{\prime} M-1\right)$ $=44$ PAPR calculations based on $16,384 \times 4=65,536$ samples while not needing any control bits related to PAPR reduction.

Overall, in terms of size of search space $\mathbf{V}$, with our simulation parameters, PSLM requires about $\frac{1}{64}$ of the complexity required by the original SLM. MSSLM requires $\frac{1}{128}$ of the complexity required by the original SLM. For the SSLM case, it only requires $\frac{1}{373}$ of the complexity needed by the original SLM. All SLM based methods do not need extra signaling overhead because of the inclusion of pilot signals. At the transmitter side, PLPT requires only $\frac{1}{32}$ of the processing power that is needed by the original SLM. PLPT outperforms SLM based methods because in an optimization interval of four MA symbols, PLPT optimizes twice.

\section{Conclusions}

This article has presented PAPR reduction schemes for carrier aggregated systems, using LTE-A standard as an 
example. Both OFDMA and SC-FDMA are considered and MIMO setup is included. The proposed MSSLM reduces the complexity and removes the signaling overhead required by the traditional SLM scheme with the introduction of the multi-symbol optimization interval together with the aid of a reference/pilot signal within that interval. Partial group selection is then introduced in PSLM as an additional means to ease the processing requirement. SSLM relieves more computation burden by reducing the size of the signal representation space in the PAPR optimization. PLPT method takes a different approach on generating different signal representation by linearly precoding two adjacent MA symbols groupwisely. PLPT would be better suited of handheld device (UL) as it offers more PAPR reduction gain and shifts some signal processing (detection of precoding) to the receiver side (base station). On the other hand, if a lower complexity receiver is needed at handheld devices, any one from MSSLM, PSLM and SSLM can be adopted for the DL. With a minor performance difference, SSLM offers the most complexity-efficient solution for both $\mathrm{UL}$ and DL. The proposed phase rotation detection at the receiver shows very reliable performance, confirming applicability of the proposed SLM based methods without affecting receiver performance. Overall, the proposed schemes provide appreciable PAPR reduction gains while requiring low complexity and no signaling overhead. Thus, they offer appealing solutions to the PAPR reduction for bandwidth-efficient carrier aggregated systems.

\section{Competing interests}

The authors declare that they have no competing interests.

Received: 30 September 2011 Accepted: 21 May 2012

Published: 21 May 2012

\section{References}

1. 3GPP TR 36.913, Requirements for further advancements for evolved universal terrestrial radio access (E-UTRA) (LTE-Advanced). Release. (Dec 2009)

2. 3GPP TS 36.211, Evolved Universal Terrestrial Radio Access (E-UTRA); physical channels and modulation. Release. (Dec 2009)

3. MM Rana, MS Islam, AZ Kouzani, Peak to average power ratio analysis for LTE systems, in IEEE ICCSN, Singapore, Singapore, 516-520 (Feb 2010)

4. T Jiangm, Y Wu, An overview: Peak-to-average power ratio reduction techniques for OFDM signals. IEEE Trans Broadcast. 54(2), 257-268 (2008)

5. SH Han, JH Lee, An overview of peak-to-average power ratio reduction techniques for multicarrier transmission. IEEE Wirel Comm Mag. 12(2), 56-65 (2005). doi:10.1109/MWC.2005.1421929

6. $\quad X L i, L J$ Cimini Jr, Effects of clipping and filtering on the performance of OFDM. IEEE Commun Lett. 2(5), 131-133 (1998)

7. L Wang, C Tellambura, A simplified clipping and filtering technique for PAR reduction in OFDM systems. IEEE Signal Process Lett. 12(6), 453-456 (2006)

8. H Ochiai, H Imai, Performance analysis of deliberately clipped OFDM signals. IEEE Trans Commun. 50(1), 89-101 (2002). doi:10.1109/26.975762

9. SB Slimane, Peak-to-average power ratio reduction of OFDM signals using pulse shaping, in IEEE GLOBECOM, San Francisco, USA, 3, 1412-1416 (2000)

10. M Tanahashi, $\mathrm{H}$ Ochiai, On the distribution of instantaneous power in single-carrier signals. IEEE Trans Wirel Commun. 9(3), 1207-1215 (2010)
11. NJ Baas, DP Taylor, Pulse shaping for wireless communication over time- or frequency-selective channels. IEEE Trans Commun. 52(9), 1477-1479 (2004). doi:10.1109/TCOMM.2004.833133

12. D Wulich, $L$ Goldfeld, Reduction of peak factor in orthogonal multicarrier modulation by amplitude limiting and coding. IEEE Trans Commun. 47(1), 18-21 (1999). doi:10.1109/26.747808

13. C Ciochina, D Castelain, D Mottier, H Sari, New PAPR-preserving mapping methods for single-carrier FDMA with space-frequency block codes. IEEE Trans Wirel Commun. 8(10), 5176-5186 (2009)

14. C Ciochina, D Mottier, D Castelain, Low PAPR space frequency block coding for multiuser MIMO SC-FDMA systems: specific issues for users with different spectral allocations. EURASIP J Adv Signal Process. 2011(1), 54-63 (2011). doi:10.1186/1687-6180-2011-54

15. SH Muller, JB Huber, OFDM with reduced peak-to-average power ratio by optimum combination of partial transmit sequences. IEEE Electron Lett. 33(5), 368-369 (1997). doi:10.1049/el:19970266

16. SG Kang, JG Kim, EK Joo, A novel subblock partition scheme for partial transmit sequence OFDM. IEEE Trans Broadcast. 45(3), 333-338 (1999). doi:10.1109/11.796276

17. T Jiang, W Xiang, PC Richardson, J Guo, G Zhu, PAPR reduction of OFDM signals using partial transmit sequences with low computational complexity. IEEE Trans Broadcast. 53(3), 719-724 (2007)

18. RW Bauml, RFH Fischer, JB Huber, Reducing the peak-to-average power ratio of multi-carrier modulation by selected mapping. IEEE Electron Lett. 32(22), 2056-2057 (1996). doi:10.1049/el:19961384

19. RJ Baxley, GT Zhou, Comparing selected mapping and partial transmit sequence for PAR reduction. IEEE Trans Broadcast. 53(4), 797-803 (2007)

20. ES Hassan, SE El-Khamy, MI Dessouky, SA El-Dolil, FE Abd El-Samie, Peak-toaverage power ratio reduction in space-time block coded multi-input multioutput orthogonal frequency division multiplexing systems using a small overhead selective mapping scheme. IET Commun. 3(10), 1667-1674 (2009). doi:10.1049/iet-com.2008.0565

21. MS Baek, MJ Kim, YH You, HK Song, Semi-blind channel estimation and PAR reduction for MIMO-OFDM system with multiple antennas. IEEE Trans Broadcast. 50(4), 414-424 (2004). doi:10.1109/TBC.2004.837885

22. S Suyama, H Adachi, H Suzuki, K Fukawa, PAPR reduction methods for eigenmode MIMO-OFDM transmission, in IEEE VTC, Barcelona, Spain, 1-5 (Apr 2009)

23. DW Lim, HS Noh, HB Jeon, JS No, DJ Shin, Multi-stage TR scheme for PAPR reduction in OFDM signals. IEEE Trans Broadcast. 55(2), 300-304 (2009)

24. JC Chen, $\mathrm{CP} \mathrm{Li}$, Tone reservation using near-optimal peak reduction tone set selection algorithm for PAPR reduction in OFDM systems. IEEE Signal Process Lett. 17(11), 933-936 (2010)

25. BS Krongold, DL Jones, PAR reduction in OFDM via active constellation extension. IEEE Trans Broadcast. 3, 258-268 (2003)

26. C Ciochina, F Buda, H Sari, An analysis of OFDM peak power reduction techniques for WiMAX systems, in IEEE ICC, Istanbul, Turkey, 4676-4681 (2006)

27. T Frank, A Klein, T Haustein, A survey on the envelope fluctuations of DFT precoded OFDMA signals, in IEEE ICC, Beijing, China, 3495-3500 (19-23 May 2008)

28. HG Myung, J Lim, DJ Goodman, Peak-to-average power ratio of single carrier FDMA signals with pulse shaping, in IEEE PIMRC, Helsinki, Finland, 1-5 (Sep 2006)

29. L Guan, T Jiang, D Qu, Y Zhou, Joint channel estimation and PTS to reduce peak-to-average-power ratio in OFDM systems without side information. IEEE Signal Process Lett. 17(10), 883-886 (2010)

30. P Yen, H Minn, CC Chong, PAPR reduction for bandwidth-aggregated OFDM and SC-FDMA systems, in IEEE WCNC, Cancun, Mexico, 1363-1368 (28-31 Mar 2011)

31. C Ciochina, D Mottier, H Sari, An analysis of OFDMA, precoded OFDMA and SC-FDMA for the uplink in cellular systems, International Workshop on Multi-Carrier Spread Spectrum, May 2007, Herrsching, Germany. Lecture Notes in Electrical Engineering. 1, 25-36 (2007). doi:10.1007/978-1-40206129-5_3

doi:10.1186/1687-1499-2012-179

Cite this article as: Yen and Minn: Low complexity PAPR reduction methods for carrier-aggregated MIMO OFDMA and SC-FDMA systems. EURASIP Journal on Wireless Communications and Networking 2012 2012:179. 